\title{
Quantification of the spread of Mycoplasma hyopneumoniae in nursery pigs using transmission experiments
}

\author{
Tom Meyns ${ }^{\mathrm{a}, *}$, Dominiek Maes ${ }^{\mathrm{a}}$, Jeroen Dewulf ${ }^{\mathrm{a}}$, Jo Vicca ${ }^{\mathrm{a}, \mathrm{b}}$, \\ Freddy Haesebrouck ${ }^{\mathrm{b}}$, Aart de Kruif ${ }^{\mathrm{a}}$ \\ ${ }^{a}$ Department of Reproduction, Obstetrics and Herd Health, Veterinary Epidemiology Unit, \\ Faculty of Veterinary Medicine, Ghent University, Salisburylaan 133, B-9820 Merelbeke, Belgium \\ ${ }^{\mathrm{b}}$ Laboratory of Veterinary Bacteriology and Mycology, Faculty of Veterinary Medicine, Ghent University, \\ Salisburylaan 133, B-9820 Merelbeke, Belgium
}

Received 6 July 2004; received in revised form 28 September 2004; accepted 11 October 2004

\begin{abstract}
Mycoplasma hyopneumoniae (M. hyopneumoniae) is present in almost all swine herds worldwide, but transmission of the pathogen through herds is not yet fully clarified. The aim of this study, performed in 2003, was to investigate and to quantify the transmission of $M$. hyopneumoniae under experimental conditions by means of an adjusted reproduction ratio $\left(R_{\mathrm{n}}\right)$. This $R_{\mathrm{n}}$-value, calculated according to the final size method, expresses the mean number of secondary infections due to one typical infectious piglet during the nursery period. The period lasted from 4 to 10 weeks of age, corresponding with the nursery period used in most European production systems. Additionally, a comparison was made between transmissions of highly virulent and low virulent isolates.

Forty-eight weaned piglets, free of $M$. hyopneumoniae, were housed in six separate pens. During 6 weeks, two animals experimentally infected with M. hyopneumoniae were housed together with six susceptible piglets. At the end of the study, the number of contact-infected animals was determined by the use of nPCR on bronchoalveolar lavage fluid. The $R_{\mathrm{n}}$-values of the highly virulent and the low virulent isolates were estimated to be $1.47(0.68-5.38)$ and $0.85(0.33-3.39)$, respectively. No significant difference between the groups was found $(P=0.53)$. The overall $R_{\mathrm{n}}$ was estimated to be 1.16 (0.94-4.08). Under the present experimental conditions, the transmission of M. hyopneumoniae,
\end{abstract}

* Corresponding author. Tel.: +329264 75 48; fax: +32 92647534 .

E-mail address: tom.meyns@ugent.be (T. Meyns). 
assessed for the first time by a reproduction ratio, shows that one piglet infected before weaning will infect on average one penmate during the nursery phase.

(C) 2004 Elsevier B.V. All rights reserved.

Keywords: Mycoplasma hyopneumoniae; Transmission; Reproduction ratio; Pig disease

\section{Introduction}

Mycoplasma hyopneumoniae (M. hyopneumoniae) is considered the primary pathogen associated with enzootic pneumonia (EP) (Goodwin et al., 1965; Ross, 1999) or porcine respiratory disease complex (Ross, 1999; Thacker et al., 1999). Both diseases cause important economic losses to the swine industry due to reduced growth rate, poor feed conversion ratio and increased susceptibility to infections with other organisms (Ross, 1999; Maes et al., 2003). At the present time, control is mostly accomplished by the use of management strategies, medication and/or vaccination (Mateusen et al., 2001), but optimal timing of the control measures remains a critical point. Knowledge of precise timing of the infection and complete clarification of the transmission of $M$. hyopneumoniae throughout the herd would make it possible to control the disease in a more efficient way.

It is known that immunologically naïve piglets between 3 and 12 weeks of age are equally susceptible to infections with M. hyopneumoniae (Piffer and Ross, 1984), and in many herds piglets become infected during the nursery period (Vicca et al., 2002; Ruiz et al., 2003). It has been shown that weaned piglets can infect each other during the nursery period (Clarck et al., 1991), but quantification of the infection rate has not yet been performed.

The spread of several viral agents like classical swine fever (Laevens et al., 1998; Dewulf et al., 2001), pseudorabies virus (De Jong and Kimman, 1994; Bouma et al., 1996) and porcine reproductive and respiratory syndrome virus (PRRSV) (Nodelijk et al., 2001) has already been quantified by means of a reproduction ratio. However, transmission experiments for the quantification of the spread of bacterial diseases have not been frequently used until today. Recently, Velthuis et al. (2003) successfully designed an experimental setting to describe the transmission of Actinobacillus pleuropneumoniae.

The purpose of this study was to investigate and quantify the transmission of $M$. hyopneumoniae under experimental conditions among weaned piglets, by calculating an adjusted reproduction ratio $\left(R_{\mathrm{n}}\right)$. Since previous work has clearly shown differences in virulence between $M$. hyopneumoniae isolates (Vicca et al., 2003), we also investigated possible differences in transmission between groups infected with a highly virulent and a low virulent $M$. hyopneumoniae isolate, respectively.

\section{Materials and methods}

\subsection{Experimental design}

Forty-eight 3-week-old cross-bred (Rattlerow Seghers ${ }^{\circledR}$, Buggenhout, Belgium) castrated male piglets were weaned and moved to the animal facilities of the Faculty 
of Veterinary Medicine, Ghent University, Belgium. The piglets were obtained from a herd that was free of M. hyopneumoniae and PRRSV. The source herd was a high health breeding herd in which repeated serological monitoring of sows and pigs of different age categories had been performed during the last 5 years. During that time, there was no clinical or serological evidence of $M$. hyopneumoniae infection, and no lung lesions typical for $M$. hyopneumoniae infection were observed in slaughter pigs. The experiment was performed in 2003. Animals were randomly assigned into two groups at arrival. The animals were fed a commercial feed without antimicrobials and were allowed to acclimatize for 1 week. At 28 days of age, within each group, six pigs (seeder pigs) were randomly selected for inoculation with a highly virulent or a low virulent $M$. hyopneumoniae isolate. Two infected animals were placed in each of the six isolated pens: three pens for the pigs inoculated with a highly virulent isolate and three pens for the pigs inoculated with a low virulent isolate. The inoculation day was designated as day 0 post-infection (0 DPI). At 2 DPI, six susceptible contact animals were added to each pen. Each group of eight piglets was housed in a pen equipped with absolute filters (HEPA U15) to avoid spread of M. hyopneumoniae and other pathogens between the groups. The disease spread was monitored during a period of 6 weeks, corresponding with the duration of the nursery period mostly applied under field conditions in Western Europe. The study was performed after approval of the Ethical Committee for Animal Experiments of the Faculty of Veterinary Medicine, Ghent University.

\subsection{M. hyopneumoniae inocula and intratracheal inoculation}

Two Belgian M. hyopneumoniae field isolates, obtained from the lungs of pigs from two different Belgian farrow-to-finish herds, were used. These isolates had been proven to be highly virulent and low virulent during earlier comparative virulence studies (Vicca et al., 2003). The highly virulent isolate (MhF7) was obtained from a herd experiencing clinical symptoms associated with EP and could be filter cloned after 10 passages in vitro. The low virulent isolate (MhF13) was obtained from an infected herd without clinical symptoms associated with EP and could be filter-cloned after nine passages in vitro.

Twelve pigs were anaesthetized intramuscularly with $0.22 \mathrm{ml} / \mathrm{kg}$ of a mixture of xylazin (Xyl-M 2\% ${ }^{\circledR}$, VMD, Arendonk, Belgium) and zolazepam and tiletamin (Zoletil $^{\circledR}$ 100, Virbac, Louvain la Neuve, Belgium) and inoculated intratracheally with $7 \mathrm{ml}$ inoculum, containing $1 \times 10^{7} \mathrm{CCU} / \mathrm{ml}$ of the highly virulent or the low virulent M. hyopneumoniae isolate. Success of inoculation was assessed by the results of seroconversion and by a nested polymerase chain reaction (nPCR) results on bronchoalveolar lavage (BAL) fluid.

\subsection{Clinical observations}

Each group of pigs was observed daily for 15 min. Body condition, appetite, presence of dyspnea and tachypnea were recorded. In addition, a clinical respiratory disease score (RDS) was assessed daily (Halbur et al., 1996). RDS could range from 0 to 6: 0 (no 
coughing), 1 (mild coughing after an encouraged move), 2 (mild coughing while leaving the pigs undisturbed), 3 (moderate coughing after encouraged move), 4 (moderate coughing while leaving the pigs undisturbed), 5 (severe coughing after encouraged move), and 6 (severe coughing while leaving the pigs undisturbed). Summation of coughing scores was made and a median respiratory score, with inter-quartile range, was calculated for the 42 days of the trial.

\subsection{Serology}

Blood samples from all pigs were taken upon arrival, at 23, 32 and 43 DPI to detect antibodies against $M$. hyopneumoniae, using the DAKO ${ }^{\circledR}$ Mh ELISA (DAKO, Glostrup, Denmark) (Feld et al., 1992). Sera with optical density values (OD-values) $<50 \%$ of the $\mathrm{OD}_{\text {buffer-control }}$ were considered positive. OD-values $>50 \%$ of the $\mathrm{OD}_{\text {buffer-control }}$ were classified as negative. The blood samples taken upon arrival and at 43 DPI were also checked for PRRSV antibodies in the serum by means of the HerdChek ${ }^{\circledR}$ PRRS ELISA (Idexx Laboratories, Westbrook, ME, USA).

\subsection{Lung lesions, immunofluorescence, histopathology and bacteriological examination}

Macroscopic and typical M. hyopneumoniae induced pneumonic lesions were quantified using a lung lesion score diagram (Hannan et al., 1982). Total lung scores could vary between score 0 (no lesions) and a theoretical maximum of 35 . A sample of the left lung was used to perform a semi-quantitative immunofluorescence assay (score 0-3) to detect the presence of M. hyopneumoniae (Kobisch et al., 1978). In addition, tissue samples of each left lung were taken for histopathological examination. Samples were collected on the edge of lung lesions, if present. In case no macroscopic lesions were present, the sample was collected from the left cranial lung lobe. The percentage of lung area occupied by air (percentage of air) was examined by means of an image analysis system (Optimas ${ }^{\circledR}$ 6.5, Media Cybernetics, Silver Spring, USA). Using light microscopy, the severity of peribronchiolar and perivascular lymphohistiocytic infiltration and nodule formation consistent with $M$. hyopneumoniae induced pneumonia lesions, were scored (Morris et al., 1995). A sample of each left lung was inoculated on Columbia agar supplemented with 5\% sheep blood (Oxoid, Hampshire, UK) with a Staphylococcus intermedius streak for support of Actinobacillus sp. growth, and on Columbia CNA agar (Oxoid, Hampshire, UK). Plates were incubated overnight in a $5 \% \mathrm{CO}_{2}$-enriched environment at $37^{\circ} \mathrm{C}$, and identification of isolated bacteria was performed as described by Quinn et al. (1994).

\subsection{BAL and $n P C R$}

All piglets were euthanized at 43 DPI by deep anaesthesia with $0.3 \mathrm{ml} / \mathrm{kg}$ of a mixture of xylazin (Xyl-M 2\% ${ }^{\circledR}$, VMD, Arendonk, Belgium) and zolazepam and tiletamin (Zoletil $^{\circledR}$ 100, Virbac, Louvain la Neuve, Belgium), followed by exsanguination. The 
lungs were removed and the BAL was performed on the right lung to detect the presence of M. hyopneumoniae organisms with a nPCR test (Stärk et al., 1998). Every right lung was washed with $50 \mathrm{ml}$ phosphate buffered saline (PBS), and the recovered fluid was centrifuged at $4000 \times g$ during $30 \mathrm{~min}$. The pellet of the lavage fluid was resuspended in $1 \mathrm{ml}$ PBS and stored at $-80{ }^{\circ} \mathrm{C}$ until DNA extraction was performed. DNA was extracted using a DNA easy Kit (Westburg, The Netherlands), and a nPCR test was performed on the extract. If BAL fluid was found positive by nPCR, the animal was considered to be infected.

\subsection{Statistical analysis}

Estimation of the transmission of $M$. hyopneumoniae in each group was done using a stochastic infection model. Therefore, we assumed that the process of transmission of $M$. hyopneumoniae among the piglets was in accordance with the susceptible-infectious $(S-I)$ model. We assumed that once an animal was infected, it did not recover before the end of the trial and remained infectious. Given that the population exists of $(S, I)$ animals, after an infection occurred, it consists of $(S-1, I+1)$ animals. In the model, the number of contact infections, determined by the number of positive BAL fluids at the end of the trial, was the observed variable $\left(X_{i}\right) . X_{i}$ is also called the 'final size' of the outbreak. Because the final size is a discrete stochastic variable, it can only attain whole numbers, and each of these has its own probability. Using the algorithm described by De Jong and Kimman (1994), we calculated the probability distribution of the final size for the given parameters and start conditions. This distribution only depended on the $R_{\mathrm{n}}$-value (Diekmann et al., 1990) and is defined as the mean number of secondary cases caused by one typical infectious pig during the nursery period. In our study, we had six populations with $N=8$ animals, where initially two animals were infectious $\left(I_{0}=2\right)$ and six animals were susceptible $\left(S_{0}=6\right)$ (non-infected contact-exposed pigs). In the experiment, the probability distribution of the final size was represented by $F\left(X_{i} \mid R_{\mathrm{n}}, N\right.$, $\left.S_{0}, I_{0}\right)$.

We used the maximum likelihood estimator (MLE) to assess the $R_{n}$-values of the highly virulent and low virulent groups. This MLE is calculated numerically from

$$
R_{\mathrm{n}, \text { mle }}=\max _{R_{\mathrm{n}}} \prod_{i=1}^{m} F\left(X_{i}, R_{\mathrm{n}} \mid N, S_{0}, I_{0}\right)
$$

in which $F\left(X_{i}, R_{\mathrm{n}} \mid N, S_{0}, I_{0}\right)$ is the likelihood function for the observed value $X_{i}$, when $N, S_{0}$ and $I_{0}$ are given and $m$ is the number of experiments.

A linear mixed effect model (S-plus 6.1), using pen as random variable, was used for the analysis of clinical symptoms and histopathological results. Lung lesion scores were log transformed and analysed using logistic regression analysis (SPSS 11.0). Time until first seroconversion was analysed by Cox regression survival analysis (SPSS 11.0). Results of inoculated pigs and contact pigs were always analysed separately. Statistical results were considered to be significant when $P$-values were lower than 0.05 . Data were analysed using the statistical packages SPSS 11.0 (SPSS Inc., IL, USA) and S-Plus 6.1 (Insightful Corp., Seattle, USA). 


\section{Results}

\subsection{Major parameters}

\subsection{1. $n P C R$ on $B A L$}

All animals experimentally infected with $M$. hyopneumoniae were positive for $M$. hyopneumoniae in the BAL by nPCR testing at 43 DPI. In the highly virulent group, 10 out of $18(56 \%)$ contact piglets were found positive for M. hyopneumoniae, while in the low virulent group, six out of $18(33 \%)$ contact animals were found positive for $M$. hyopneumoniae. The distribution in each pen of contact piglets that became contactinfected is shown in Table 1.

\subsubsection{Reproduction ratio}

The $R_{\mathrm{n}}$-value (95\% confidence interval) was $1.47(0.68-5.38)$ and $0.85(0.33-3.39)$ for animals infected with the highly virulent and the low virulent isolates, respectively. Since no significant difference was found between the $R_{\mathrm{n}}$-value in these two groups $(P=0.53)$, the overall $R_{\mathrm{n}}$-value was calculated, by combining the outcomes of all the six transmission experiments. This overall $R_{\mathrm{n}}$-value was $1.16(0.94-4.08)$.

\subsection{Minor parameters}

\subsubsection{Clinical symptoms}

Pigs had their first positive coughing score on average at 14.96 DPI, with a spread of 4 31 DPI. All piglets had at least 1 day with a positive coughing score. First positive coughing score was noted on average at 14.56 DPI (5-28 DPI) and at 18.89 DPI (11-31 DPI), for contact pigs in the highly virulent and the low virulent groups, respectively. Piglets inoculated with the highly virulent isolate had a first positive coughing score at $6.83 \mathrm{DPI}$ (4-10 DPI), while animals inoculated with the low virulent isolate had the first coughing score later, at $10.83 \mathrm{DPI}(7-13 \mathrm{DPI})$.

The median (interquartile range) respiratory score for the inoculated animals was 85.0 (66.5-136.5) and 34.0 (19.5-40.5) in the highly virulent and in the low virulent groups, respectively $(P<0.05)$. The median respiratory score for the contact pigs was $16.0(9.8-$ $23.3)$ and $8.0(6.0-12.3)$ in the highly virulent and in the low virulent groups, respectively $(P>0.05)$.

\section{Table 1}

Distribution of infection ${ }^{\mathrm{a}}$ in six different nursery pens measured by $\mathrm{nPCR}$ in $\mathrm{BAL}^{\mathrm{b}}$ fluid in a Belgian study of transmission of M. hyopneumoniae, 2003

\begin{tabular}{|c|c|c|c|c|c|}
\hline \multicolumn{6}{|c|}{ No. of infected animals/no. of susceptible animals } \\
\hline \multicolumn{3}{|c|}{ Highly virulent $M$. hyopneumoniae } & \multicolumn{3}{|c|}{ Low virulent $M$. hyopneumoniae } \\
\hline Pen 1 & Pen 2 & Pen 3 & Pen 4 & Pen 5 & Pen 6 \\
\hline $2 / 6$ & $3 / 6$ & $5 / 6$ & $2 / 6$ & $2 / 6$ & $2 / 6$ \\
\hline
\end{tabular}


Table 2

Number of nursery pigs (\%) serologically ${ }^{\mathrm{a}}$ positive for $M$. hyopneumoniae at different days post-infection in a Belgian transmission study, 2003

\begin{tabular}{llll}
\hline & \multicolumn{2}{l}{ Days post-infection } & \\
\cline { 2 - 4 } & 23 & 32 & 43 \\
\hline Highly virulent isolate & $4(67 \%)$ & $5(83 \%)$ & $5(83 \%)$ \\
$\quad$ Inoculated $(n=6)$ & 0 & 0 & $2(11 \%)$ \\
$\quad$ Contact pigs $(n=18)$ & 0 & $1(17 \%)$ & $4(67 \%)$ \\
Low virulent isolate & 0 & 0 & 0 \\
$\quad$ Inoculated $(n=6)$ & & 0 & \\
Contact pigs $(n=18)$ &
\end{tabular}

${ }^{\text {a }}$ Assessed by use of DAKO ${ }^{\circledR}$ Mh ELISA at 23, 32 and 43 days post-infection.

One of the susceptible piglets in the highly virulent group had symptoms of meningitis. This piglet was treated with amoxycillin and non-steroidal anti-inflammatory drugs and recovered after treatment. No other piglets showed clinical symptoms apart from coughing.

\subsubsection{Serology}

Upon arrival, all piglets were serologically negative for M. hyopneumoniae and PRRSV. The results of the serological examination for M. hyopneumoniae at 23, 32 and 43 DPI are presented in Table 2. At the end of the study, none of the piglets had antibodies against PRRSV. Although highly virulent isolates induced an earlier seroconversion, with lower OD-values (data not shown) compared with the low virulent isolates, time until first seroconversion was not significantly different between both inoculated groups $(P=0.147)$.

\subsubsection{Lung lesion scores}

The mean lung lesion scores of the inoculated pigs were 8.50 and 1.93 for animals experimentally infected with a highly virulent isolate and a low virulent isolate, respectively $(P>0.05)$. The mean lung lesion scores of the contact animals were 0.61 and 0.18 in the highly virulent and low virulent groups, respectively $(P>0.05)$. These scored lung lesions, red to dark red consolidations, were typically for a $M$. hyopneumoniae infection. Five out of the 18 contact piglets in the highly virulent group and four of 18 contact piglets in the low virulent group had macroscopic lung lesions. Besides lesions indicative for $M$. hyopneumoniae infection, one contact-infected piglet had pleuritis in the low virulent group, and five out of 24 animals had pleuritis and/or pericarditis in the highly virulent group.

\subsubsection{Bacteriology}

In the highly virulent group, Streptococcus suis (S. suis) was isolated from the lungs of 19 of the 24 animals and Actinobacillus minor (A. minor) from the lungs of one animal. In the low virulent group, $S$. suis was isolated from the lungs of 16 of the 24 animals, $A$. minor from the lungs of two animals and Pasteurella multocida from the lungs of one animal.

\subsubsection{Immunofluorescence and histology}

$M$. hyopneumoniae was demonstrated by immunofluorescence testing in the lungs of five of the six pigs intratracheally inoculated with the highly virulent isolate, and in one of the six pigs intratracheally inoculated with the low virulent isolate. Only one out of the 18 
contact pigs in each group was found positive. The average scores of infiltration of inflammatory cells around respiratory airways and blood vessels were 1.00 and 0.73 for the inoculated pigs in the highly virulent and low virulent groups, respectively $(P>0.05)$. The percentages of air in the lungs were 55.70 and 52.63 for inoculated pigs in the highly virulent and the low virulent groups, respectively $(P>0.05)$.

\section{Discussion}

The purpose of this study was to measure the spread of $M$. hyopneumoniae in nursery pigs and to calculate an adjusted reproduction ratio $\left(R_{\mathrm{n}}\right)$ based on the outcome of the transmission experiments. It is believed that once infection is established in a few pigs, transmission among penmates occurs, especially after animals are pooled together at weaning time (Ross, 1999). According to what was expected based on the results of previous experiments (Etheridge et al., 1979) and based on data from field observations (Vicca et al., 2002), we were able to demonstrate transmission in all experimental groups during the nursery period. $R_{\mathrm{n}}$-values can certainly be used to evaluate differences in spread between groups with a different treatment (e.g. vaccination or medication) or to evaluate the influence of certain control strategies on the spread of M. hyopneumoniae.

One of the main problems in transmission experiments for bacterial diseases is to determine the exact duration of the infectious period, because diagnostic tools are limited to define the onset and the end of an infectious period for most bacteriological infections and especially for $M$. hyopneumoniae. Therefore we calculated an adjusted reproduction ratio. This $R_{\mathrm{n}}$-value differed from the conventional basic reproduction ratio $\left(R_{0}\right)$ because we did not take into account the entire infectious period, but only a strictly limited observation period. This was done because it was not possible to determine the onset and the end of the infectious period of animals infected with $M$. hyopneumoniae in our trial. In this experiment we only used the infectious time during the nursery period and therefore we could not be sure that the entire infectious period was included. In consequence, the calculated reproduction ratio is only valid for this specified period. The nursery period is particularly important because a few animals, which became infected before weaning, could infect a considerable number of other piglets during this period. In that way, several piglets become infected with $M$. hyopneumoniae before the finishing period, which might result in long-lasting and considerable economic losses attributed to mycoplasmal pneumonia (Maes et al., 2003). In our model, an $R_{\mathrm{n}}$-value of 1 means that one animal, infected before the nursery period, will result on average in one contact-infected animal during the nursery period. The overall $R_{\mathrm{n}}$-value of 1.16 indicates that the spread of $M$. hyopneumoniae is not excessive, but in general the infection will be maintained and will even result in more infectious animals at the end of the nursery period. Based on positive findings of M. hyopneumoniae in sows up to the seventh parity (Calsamiglia and Pijoan, 2000), it is reasonable to expect that fattening pigs infected with M. hyopneumoniae will remain infected during the rest of the fattening period and might infect other animals.

The difference in transmission between the highly virulent and the low virulent isolates was not statistically significant, but there was a tendency towards a more intensive spread in the highly virulent group. We used 48 piglets in the present experiment, but to find a 
significant difference, more pigs should be used in this experiment. The difference in spread is possibly due to a higher load of mycoplasmas in the respiratory tract of these animals. Although this hypothesis was not investigated, the faster seroconversion after inoculation and the more intense immunofluorescence also point to a more effective multiplication in the lungs of the highly virulent $M$. hyopneumoniae strain. The results concerning clinical, macroscopical and serological investigations were necessary to confirm that we really do deal with two strains of different virulence. These results of the present study confirmed the results of previous experiments (Vicca et al., 2003) concerning the difference of the highly virulent and the low virulent strains, but the number of inoculated animals was too limited to find significant results for all parameters.

We used the positive results in the BAL fluid for the calculation of the $R_{\mathrm{n}}$ because this is believed to be the most sensitive parameter to predict an infection with $M$. hyopneumoniae at individual level (Kurth et al., 2002). Nasal swabs were not used since results are less reliable. The nasal cavity is not the natural location for $M$. hyopneumoniae, so it is possible to obtain negative nasal swabs, even if infectious $M$. hyopneumoniae is present in the lungs. On the other hand, it is also possible to obtain positive nasal swabs, because only mycoplasmal DNA and not viable or infectious $M$. hyopneumoniae organisms are detected with PCR. Serological diagnosis relies upon the response of animals to the organism, and after experimental infection, pigs show positive antibody response with DAKO $^{\circledR} \mathrm{Mh}$ ELISA after 2-8 weeks (Sörensen et al., 1997). This highly variable and extended period is not accurate enough to predict the infection status in this experiment. This is also clear in our results where only $75 \%$ of the inoculated pigs had seroconverted by the end of the trial.

The design of our study differs in several ways from the situation occurring in commercial pig herds. Firstly, the experimental infection occurred as late as 2 days before the start of the experimental period. The inoculated animals became fully infectious after 1 or 2 weeks (Sörensen et al., 1997). In situations where piglets become infected during the first days of their life, they will be fully infectious during the whole nursery period and this will possibly result in a higher transmission. Also the volume of air available per pig is mostly smaller in commercial herds compared with the situation in this experimental design, which could possibly have led to a lower transmission in our experiment. On the other hand, the seeder pigs were inoculated with a relatively high dose of $M$. hyopneumoniae organisms. This high dose might not be reached under natural conditions and could have increased the transmission. The fact that only eight animals were used in one experimental unit presumably did not influence the $R_{\mathrm{n}}$-values since the transmission was calculated taking into account the initial number of infectious and susceptible animals in the experiment (Diekmann et al., 1990). In a transmission experiment with pseudorabies virus, Bouma et al. (1995) showed that there was no difference in the estimated transmission ratio based upon experiments with different numbers of infectious and susceptible animals at the start of the study.

\section{Conclusion}

This is the first time that reproduction ratios are calculated for M. hyopneumoniae. Based upon the findings in this experiment, we can conclude that, under the present 
experimental conditions, pigs infected with M. hyopneumoniae will infect on average one penmate during the nursery period.

\section{Acknowledgements}

This research was supported by The Institute for the Promotion of Innovation through Science and Technology in Flanders (IWT Vlaanderen: grant no. 21141).

\section{References}

Bouma, A., de Jong, M., Kimman, T., 1995. Transmission of pseuodrabies virus within pig populations is independent of the size of the population. Prev. Vet. Med. 23, 163-172.

Bouma, A., de Jong, M., Kimman, T., 1996. Transmission of two pseudorabies virus strains that differ in virulence and virus excretion in groups of vaccinated pigs. Am. J. Vet. Res. 57, 43-47.

Calsamiglia, M., Pijoan, C., 2000. Colonisation state and colostral immunity to Mycoplasma hyopneumoniae of different parity sows. Vet. Rec. 146, 530-532.

Clarck, L., Armstrong, C., Scheidt, A., Sands-Freeman, L., Knox, K., 1991. Investigating the transmission of Mycoplasma hyopneumoniae in a swine herd with enzootic pneumonia. Vet. Med. 86, 543-550.

De Jong, M., Kimman, T., 1994. Experimental quantification of vaccine-induced reduction in virus transmission. Vaccine 12, 761-766.

Dewulf, J., Laevens, H., Koenen, F., Mintiens, K., de Kruif, A., 2001. An E2 sub-unit marker vaccine does not prevent horizontal or vertical transmission of classical swine fever virus. Vaccine 20, 86-91.

Diekmann, O., Heesterbeek, J., Metz, J., 1990. On the definition and computation of the basic reproduction ratio $R_{0}$ in models for infectious diseases in heterogeneous populations. J. Math. Biol. 28, 365-382.

Etheridge, J., Cottew, G., Lloyd, L., 1979. Isolation of Mycoplasma hyopneumoniae from lesions in experimentally infected pigs. Aust. Vet. J. 55, 356-359.

Feld, N., Qvist, P., Ahrens, P., Friis, N., Meyling, A., 1992. A monoclonal blocking ELISA detecting serum antibodies to Mycoplasma hyopneumoniae. Vet. Microbiol. 30, 35-46.

Goodwin, R., Pomeroy, A., Whittlestone, P., 1965. Production of enzootic pneumoniae in pigs with mycoplasma. Vet. Rec. 77, 1247-1249.

Halbur, P., Paul, P., Meng, X., Lum, M., Andrews, J., Rathje, J., 1996. Comparative pathogenicity of nine US porcine reproductive and respiratory syndrome virus (PRRSV) isolates in a 5-week-old cesarean-derived, colostrum-deprived pig model. J. Vet. Diagn. Invest. 8, 11-20.

Hannan, P., Bhogal, B., Fish, J., 1982. Tylosine tartrate and tiamutilin effects on experimental piglet pneumonia induced with pneumonic pig lung homogenate containing mycoplasmas, bacteria and viruses. Res. Vet. Sci. 33, 76-88.

Kobisch, M., Tillon, J., Vannier, Ph., Magueur, S., Morvan, P., 1978. Pneumonie enzootique à Mycoplasma suipneumoniae chez le porc: diagnostic rapide et recherché d'anticorps. Res. Méd. Vet. 154, 847-852.

Kurth, K., Hsu, T., Snook, E., Thacker, E., Thacker, B., Minion, C., 2002. Use of a Mycoplasma hyopneumoniae nested polymerase chain reaction test to determine the optimal sampling sites in swine. J. Vet. Diagn. Invest. 14, 463-469.

Laevens, H., Koenen, F., Deluyker, H., Berkvens, D., de Kruif, A., 1998. An experimental infection with classical swine fever virus in weaner pigs. I. Transmission of the virus, course of the disease, and antibody response. Vet. Quart. 20, 41-45.

Maes, D., Verbeke, W., Vicca, J., Verdonck, M., de Kruif, A., 2003. Benefit to cost of vaccination against Mycoplasma hyopneumoniae in pig herds under Belgian market conditions from 1996 to 2000. Livest. Prod. Sci. 83, 85-93.

Mateusen, B., Maes, D., Hoflack, G., Verdonck, M., de Kruif, A., 2001. A comparative study of the preventive use of tilmicosin phosphate (pulmotil premix) and Mycoplasma hyopneumoniae vaccination in a pig herd with chronic respiratory disease. J. Vet. Med. B 48, 733-741. 
Morris, C., Gardner, I., Hietala, S., Carpenter, T., 1995. Enzootic pneumonia: comparison of cough and lung lesions as predictors of weight gain in swine. Can. J. Vet. Res. 59, 197-204.

Nodelijk, G., de Jong, M., van Leengoed, L., Wensvoort, G., Pol, J., Steverink, P., Verheijden, J., 2001. A quantitative assessment of the effectiveness of PRRSV vaccination in pigs under experimental conditions. Vaccine 19, 3636-3644.

Piffer, I., Ross, R., 1984. Effect of age on susceptibility of pigs to Mycoplasma hyopneumoniae pneumonia. Am. J. Vet. Res. 45, 478-481.

Quinn, P., Carter, M., Markey, B., Carter, G. (Eds.), 1994. Clinical Veterinary Microbiology. Mosby, Edinburgh, pp. 254-259.

Ross, R., 1999. Mycoplasmal diseases. In: Leman, A.D., Straw, B.E., D’Allaire, S., Mengeling, W.L., Taylor, D.J. (Eds.), Diseases of Swine. 8th ed. The Iowa State University Press, Ames, IA, pp. 495-509.

Ruiz, A., Utrera, V., Pijoan, C., 2003. Effect of Mycoplasma hyopneumoniae sow vaccination on piglet colonization at weaning. J. Swine Health Prod. 11, 131-135.

Sörensen, V., Ahrens, P., Barfod, K., Feenstra, A., Feld, N., Friis, N., Bille-Hansen, V., Jensen, N., Pedersen, M., 1997. Mycoplasma hyopneumoniae infection in pigs: duration of the disease and evaluation of four diagnostic assays. Vet. Microbiol. 54, 23-34.

Stärk, K., Nicolet, J., Frey, J., 1998. Detection of Mycoplasma hyopneumoniae by air sampling with a nested PCR assay. Appl. Environ. Microbiol. 64, 543-548.

Thacker, E., Halbur, P., Ross, R., Thanawongnuwech, R., Thacker, B., 1999. Mycoplasma hyopneumoniae potentiation of porcine reproductive and respiratory syndrome virus-induced pneumonia. J. Clin. Microbiol. 37, 620-627.

Velthuis, A., de Jong, M., Kamp, E., Stockhofe, N., Verheijden, J., 2003. Design and analysis of an Actinobacillus pleuropneumoniae transmission experiment. Prev. Vet. Med. 60, 53-68.

Vicca, J., Maes, D., Thermote, L., Peeters, J., Haesebrouck, F., de Kruif, A., 2002. Patterns of Mycoplasma hyopneumoniae infections in Belgian farrow-to-finish pig herds with diverging disease-course. J. Vet. Med. B 49, 349-353.

Vicca, J., Stakenborg, T., Maes, D., Butaye, P., Peeters, J., de Kruif, A., Haesebrouck, F., 2003. Evaluation of virulence of Mycoplasma hyopneumoniae field isolates. Vet. Microbiol. 97, 177-190. 